\title{
New ways with solar neutrinos
}

\section{The discrepancy between predicted and measured neutrinos from the Sun is an embarrassment. Now there are two schemes for improving on past measurements. They deserve serious backing.}

MuCH of the present excitement in cosmology rests on the recognition that there must be a close connection between this conceptually esoteric field and high-energy particle physics, the stamping-ground of those who would push experimental measurement to the limit. The result is that people are always speaking in measured tones about the common ground between the very large and the very small.

And there are successes on the record. Astrophysics shows the neighbourhood of a neutron star to be a kind of natural highenergy physics laboratory. The abundance of deuterium relative to hydrogen in the Universe now is almost exactly that calculated from the assumption that the big bang was a time when particles now unstable were predominant in the Universe. And the temperature of the microwave background, $2.7 \mathrm{~K}$, while it is explicable in other ways, is consistent with the view that the whole world was once a high-energy physics free-for-all. So is it not at least a minor scandal that there persists such uncertainty about the Sun's output of neutrinos? Especially when the energies supposedly carried by those particles are measured in $\mathrm{MeV}$, not the $\mathrm{GeV}$ which are now the common coinage of the particle physicists?

It is now more than a decade since $\mathbf{R}$. Davis $\mathrm{Jr}$ reported the first results with his unique neutrino detector, essentially a huge tank containing 380 cubic metres of tetrachloroethylene buried deep in the Homestake Mine in Colorado. The simple objective was to count the number of chlorine-37 nuclei converted into argon-37 by interaction with a neutrino (and the subsequent loss of a negative electron). The difficulty, ten years ago, was that the rate of conversion and thus the inferred intensity of the neutrino flux was almost an order of magnitude less than expected from what is known of the nuclear reactions in the Sun. All this is now part of the general lore.

Not much has happened since. Or, rather, there has been much talk and speculation but very little experiment. For a time, there was some hope that the discrepancy might be explained away on the basis of the experiments carried out by F. W. Reines at the University of California, Irvine, whose reports of neutrino "oscillations", the quantum mixing together of different types of neutrinos to form the neutrinos of the real world, also bore on the possibility that neutrinos may have a non-zero mass.

But that line of thought has become unfashionable. The experiments are not easy to repeat, and in any case the cosmologists no longer hope to close the Universe with neutrinos of small mass (a fraction of that of the electron). And while the Davis measurement may be reconciled with expectation by using extremes of several astrophysical parameters, leading in particular to a low core temperature, that seems an ungainly way out of the difficulty.

So what is there left to do? Simply elaborating the Davis measurement would be unprofitable, with a rate of conversion of chlorine into argon only barely above the background. J. Bahcall at Princeton has for the past six years been canvassing the use of gallium-71 as a detector isotope. He would buy a stock of several tons of gallium metal, bury in a mine for several years and then sell it back to the original owners. That device would have the virtue of being sensitive to the least energetic and most abundant neutrinos from the Sun, those produced by the direct interaction of protons. One of these fiscal years, the US Congress may look kindly at the project.

Meanwhile Davis (at the Brookhaven National Laboratory) and a group of colleagues scattered over the United States have suggested yet another neutrino detector. The principle is again the same convert an isotope of one element into an isotope of another by neutrino interaction, making sure that scores or hundreds of atoms of the product can be measured. The new plan is to convert bromine- 81 into krypton-81 (again by the absorption of a neutrino and the loss of an electron). But this time, the product is only weakly radioactive (with a half-life of 200,000 years).

In principle, the experiment (if ever carried out) would have the advantage of being neatly complementary to that proposed by Bahcall. Indeed, bromine-81 nuclei would interact only with neutrinos whose energy exceeded the threshold of 470 $\mathrm{keV}$, which implies that they would be converted into krypton nuclei chiefly by neutrinos arising within the Sun from the radioactive decay of boron-7.

The hope that the quantities of krypton-81 produced can be measured will stretch most people's credulity. If the calculations are correct, krypton isotope atoms should accumulate in the 380 cubic metres of the tank (supposed filled with an organic compound of bromine) at a rate of about two a day, or some hundreds every six months. How are such small quantities of a stable isotope to be detected?

Obviously it helps that krypton is a rare gas, essentially insoluble in organic liquids. Indeed, using their tank filled with its organochlorine liquid, Davis and his group have been able to test the feasibility of what they propose. Having a system for the extraction of argon from the tank, they can just as easily isolate krypton. They find that leakage of atmospheric krypton into the system should not be a serious source of whatever krypton- 81 might be produced by neutrino conversion. After say a year, their bromine tank might contain 1,000 atoms of artificial krypton-81.

But how to measure such tiny amounts? Perhaps because they are anxious not to discourage potential backers, the authors make light of the difficulties in their account of a simulation they have carried out (G.S. Hurst et al. Phys.Rev.Lett. 53, $1116 ;$ 1984).

The technique consists merely of repetitive mass spectroscopy. Atoms of krypton-81 in what is supposed to be a mixture of krypton isotopes are selectively ionized by a suitable laser and then accelerated selectively and embedded in a silicon target. Since the ionization process will not have been entirely selective, the embedded atoms can be recovered by heating (again with a laser) and put through the process a second time. And, if necessary, a third. Finally, the atoms are embedded in a copper/beryllium target.

Whatever the likelihood that the technique will eventually settle the problem of solar neutrinos, it seems certain to be used widely in other connections. What the authors show, however, is that starting with a sample containing 1,000 atoms of krypton-81 and the contamination expected in a neutrino experiment, they should be able to count as few as 300 atoms. Laser improvements already foreseen should reduce the limit to about 100 atoms.

For many people, this would be sufficient. But plainly Davis and his colleagues intend a renewed assault on the measurement of solar neutrinos. Indeed, they talk of the design of their complementary detectors as the first steps in the spectroscopy of solar neutrinos. They should be given a chance to put their scheme to work and save the nexus of cosmology and physics from the embarrassment on its doorstep.

John Maddox 